\title{
Heitler-Matthews model with leading-particle effect
}

\section{Stefan Grimm, Ralph Engel*, and Darko Veberič,}

Institute for Nuclear Physics, Karlsruhe Institute of Technology $\mathbf{Q} \mathbf{K I T}$, Karlsruhe, Germany

E-mail: ralph.engel@kit.edu
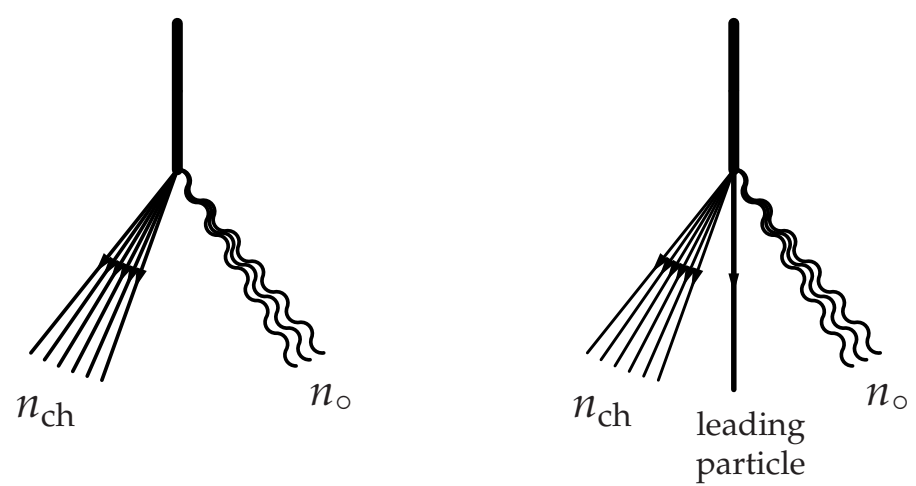

It is well known that the Heitler model and its extensions (like the Heitler-Matthews model) describe qualitatively many fundamental properties of extensive air showers initiated in the atmosphere by the high-energy cosmic rays. Typically, only the secondary particle multiplicity and the fraction of neutral-to-charged pions are considered as analytically treatable parameters of hadronic interactions. Other relevant parameters of hadronic interactions such as inelasticity and the lifetime of the leading particle are taken into account only in detailed Monte Carlo simulations. Here we present an extension of the Heitler-Matthews model that includes the leading particle effect and analytically derive the number of muons produced in air showers in a selfconsistent way. In a second step we apply this model to calculate the dependence of the depth of the shower center-of-gravity on various model parameters. In this way, we obtain predictions for the depth of shower maximum, which is a well known observable sensitive to the mass of primary particles and is regularly used in cosmic-ray research.

35th International Cosmic Ray Conference - ICRC2017

10-20 July, 2017

Bexco, Busan, Korea

${ }^{*}$ Speaker. 


\section{Introduction}

Despite the growing computing power available for the computation of simulations, understanding the physics of extensive air showers remains a very difficult problem. If one wants to have large statistics or if a large parameter space is to be explored, it is impractical to simulate the full showers at highest energies as the number of particles is too large.

Various approaches to solving this problem exist, among these are thinning algorithms [1,2], which reduce the number of particles whose trajectories and interactions have to be simulated, or hybrid simulations [3-5]. Still, even with these methods it is difficult to study large parameter spaces and to understand the physics origin of possible correlations. While detailed simulations provide the most accurate predictions for air shower nowadays, it is, in general, not possible to derive analytical relations between input and output parameters from them.

On the other hand, although numerically not reliable, analytic cascade models like the HeitlerMatthews model [6] provide very useful insights in physics relations at the heart of cascades. The simplicity of such models is well-suited for analytical calculations of shower observables, which make it possible to study parameter relations very easily. Moreover, cascade models are very illustrative and give often an fundamental understanding of the important processes at work in air showers.

The predictions of the Heitler-Matthews model are qualitatively surprisingly accurate despite the many simplifying assumptions made in the model. Motivated by this success, various extensions have been developed since the publication of the original model in 2005. These include, for example, the approximate treatment of energy-dependent secondary particle multiplicities [7] or the treatment of the density profile of the atmosphere [8]. And already in the original publication [6], an approximative attempt was made to include elasticity effects into the model, but a complete and self-consistent Heitler-Matthews model with leading-particle effects was not developed.

In this work we introduce an extended Heitler-Matthews model that takes into account the two most important features of the hadronic leading particle effect. These are the asymmetric energy distribution (often parametrized by the elasticity of an interaction) and the possibility of the immediate decay of the leading particle. In this model it is possible to calculate the number of muons and the depth of shower maximum, the latter by using the shower center of gravity, in a self-consistent way. As application we explore the dependence of these typical shower observables on features of hadronic interactions. The detailed derivation of the results given here and additional applications will be published in elsewhere [9].

Heitler-Matthews model. The Heitler-Matthews model is a very simple model which helps to understand the development of the hadronic cascade of air showers and which makes predictions for the mean values of observables $[6,10]$.

When a cosmic ray enters the atmosphere, it interacts with a nucleus and creates new particles, which in turn interact again. The assumption of the Heitler model is that particles interact after traversing one interaction length $\lambda_{\mathrm{h}}$ and that in each interaction $n_{\text {tot }}$ particles are created ( $n_{\mathrm{ch}}$ charged pions and $n_{0}$ neutral pions). This interaction model is shown on the left side of the schematic above the Abstract. The neutral pions decay into electromagnetic particles and the charged pions interact again until they decay into muons when their energy drops below the critical 


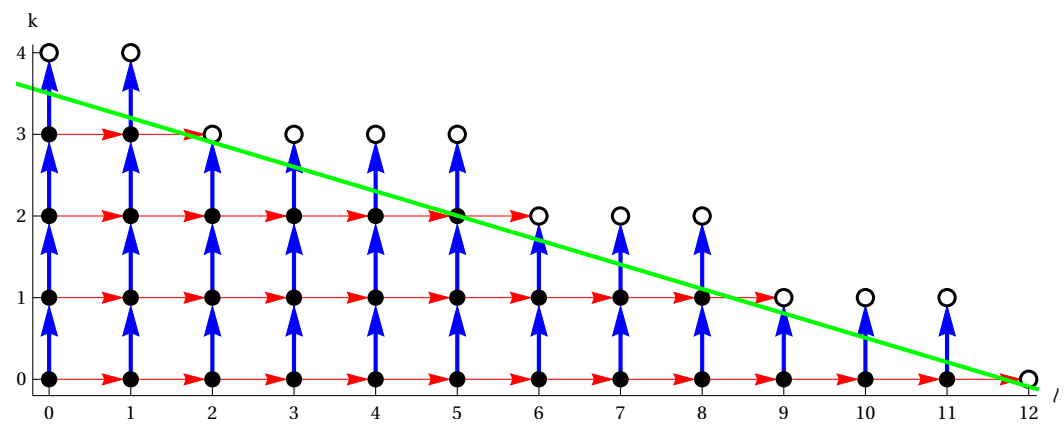

Figure 1: Shower evolution in the $\ell k$-plane. Thin red arrows denote leading branches while thick blue arrows stand for trailing branches that feed the sub-showers (black dots). The diagonal line represents the energy threshold below which sub-showers decay into muons (empty circles). $L$ and $T$ are the intersections of the decay line with the $\ell$ and $k$ axis, respectively.

energy $E_{\mathrm{c}}^{\pi}$. To allow for an analytical solution of the model, $\lambda_{\mathrm{h}}, n_{\mathrm{tot}}=n_{\mathrm{ch}}+n_{0}, f_{\mathrm{ch}}=n_{\mathrm{ch}} / n_{\mathrm{tot}}$, and $E_{\mathrm{c}}^{\pi}$ are assumed to be constant throughout the whole cascade and do not depend on the depth of the evolution.

The number of charged pions after $n$ generations is simply $n_{\mathrm{ch}}^{n}$, energy of the particles after $n$ generations is $E_{\circ} / n_{\mathrm{tot}}^{n}$. Reaching the critical energy $E_{\mathrm{c}}^{\pi}$ at generation $n_{\mathrm{c}}=\ln \varepsilon_{\circ} / \ln n_{\text {tot }}$ with $\varepsilon_{\circ}=$ $E_{\circ} / E_{\mathrm{c}}^{\pi}$, charged pions decay into muons, thus the number of muons is $N_{\mu}=n_{\mathrm{ch}}^{n_{\mathrm{c}}}=\varepsilon_{\circ}^{\gamma}$, where $\gamma=\ln n_{\mathrm{ch}} / \ln n_{\text {tot }}=1+\ln f_{\mathrm{ch}} / \ln n_{\text {tot }}$. Since $1 / n_{\text {tot }}<f_{\text {ch }}<1$ the muon elongation rate $\gamma$ can only be $0<\gamma<1$. The prediction of this model thus is that the energy dependence of the number of muons is a power law, which is what is observed in experimental data.

Asymmetric extension. In the following we want to develop an extended Heitler-Matthews model which takes the leading particle effect into account. We assume that in each interaction of a particle with the nuclei of air $n_{\text {tot }}$ particles are created: one leading particle that carries away fraction $\alpha=E_{\text {lead }} / E$ of the energy of the incoming particle $E$ and can decay into electromagnetic particles with probability $1-p$, and $n_{\text {tot }}-1$ trailing particles that get equal shares of the remaining energy, that is, they each end up with a fraction $\beta=E_{\text {trail }} / E=(1-\alpha) /\left(n_{\text {tot }}-1\right)$. Out of these $n_{\text {tot }}-1$ trailing particles only the $n_{\mathrm{ch}}$ charged particles continue to feed the hadronic shower and the remaining $n_{\circ}=n_{\text {tot }}-1-n_{\mathrm{ch}}$ feed the electromagnetic shower. This interaction model is shown on the right side of the schematic above the Abstract.

\section{Number of muons}

After a certain number of generations $n$, the energy of each individual particle in the hadronic shower is uniquely determined by its ancestry, that is, by the respective numbers of leading $(\ell)$ and non-leading (trailing) $(k)$ branches found in their genealogy, where $\ell+k \equiv n$. This energy can be derived from the particle multiplication $n_{\mathrm{tot}}^{n}=\left(1+n_{\mathrm{ch}}+n_{\circ}\right)^{n}$ and energy conservation $\left(\alpha+n_{\mathrm{ch}} \beta+n_{\circ} \beta\right)^{n}$ expressions. Furthermore, we can sort particles at generation $n$ of the hadronic cascade into sub-showers with energy $E_{\ell k}=E_{\circ} \alpha^{\ell} \beta^{k}$ where the potential number of particles in this sub-shower is $N_{\ell k}^{\prime}=\left(\begin{array}{c}\ell+k \\ \ell\end{array}\right) n_{\mathrm{ch}}^{k}$. Nevertheless, the leading particle survives only with probability $p$ so that the mean number of particles in the sub-shower is $N_{\ell k}=\left(\begin{array}{c}\ell+k \\ \ell\end{array}\right) p^{\ell} n_{\mathrm{ch}}^{k}$. Note that particles in 

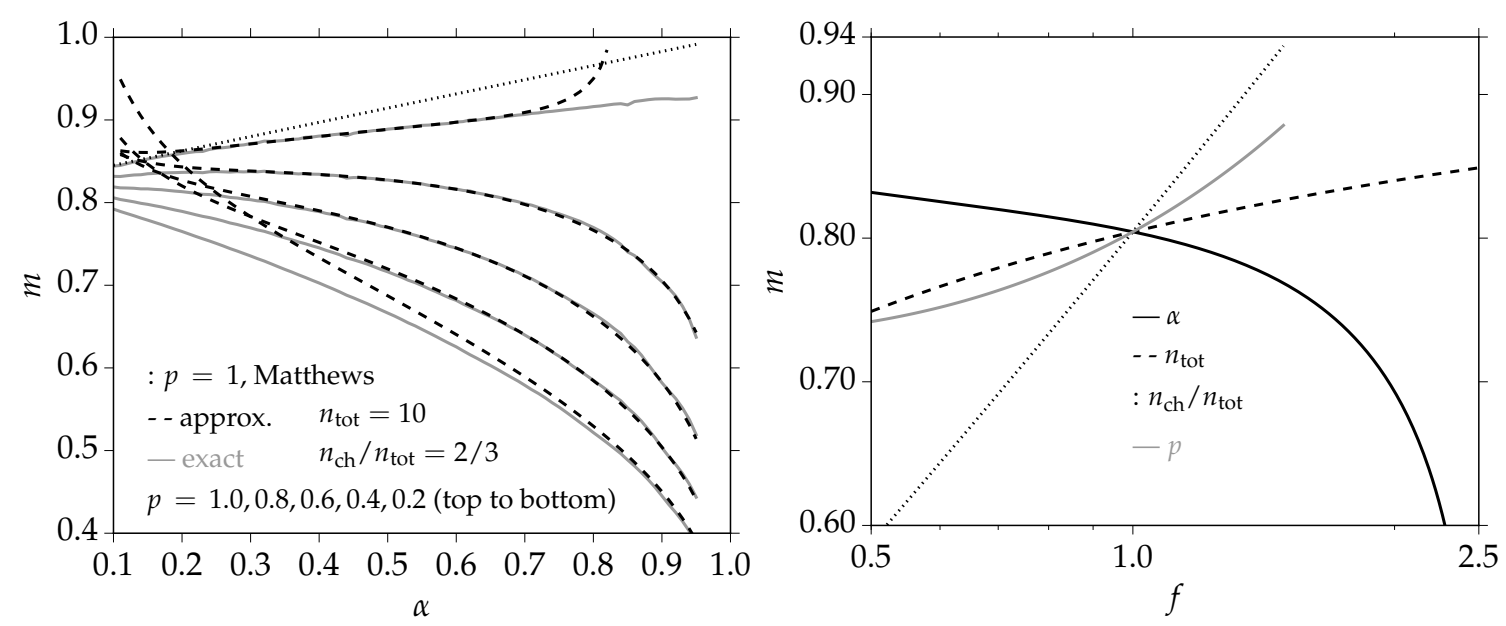

Figure 2: Left: Comparison of the exact results for the elasticity-dependence of the power-law exponent derived from fitting to the exact summation (grey curves) with the exponent derived from the calculation for different survival probabilities for the leading particle (dashed curves). We can see that the approximation breaks down if many leading particles decay, that is, if $p$ is small. The results of Matthews' approximation are shown in the dotted curve. Right: Parameter dependencies of the power-law exponent in our model. The standard values used in our model $\left(\kappa=0.4, n_{\text {tot }}=10, p=f_{\text {ch }}=2 / 3\right)$ are scaled with a factor $f$.

sub-shower $\{\ell, k\}$ have been produced either by leading branch of the parent sub-shower $\{\ell-1, k\}$ (red arrows in Fig. 1) or by the trailing branches of the $\{\ell, k-1\}$ parent sub-shower (blue arrows in Fig. 1). We can verify that indeed $1 \times N_{(\ell-1) k}+n_{\mathrm{ch}} N_{\ell(k-1)}=\left(\begin{array}{c}\ell-1+k \\ \ell-1\end{array}\right) n_{\mathrm{ch}}^{k}+n_{\mathrm{ch}}\left(\begin{array}{c}\ell+k-1 \\ \ell\end{array}\right) n_{\mathrm{ch}}^{k-1} \equiv$ $\left(\begin{array}{c}\ell+k \\ \ell\end{array}\right) n_{\mathrm{ch}}^{k}$, since any number in Pascal's triangle is a sum of the two proceeding numbers. When the energy falls below a certain critical energy $E_{\mathrm{c}}^{\pi}$, the decay of pions becomes more important than re-interaction. In our simplistic model, the charged particles below critical energy immediately decay into muons. From the particle energy we can derive critical conditions for $\ell$ and $k$, $-\ln \varepsilon_{\circ}=\ell \ln \alpha+k \ln \beta$, where $\varepsilon_{\circ}=E_{\circ} / E_{\mathrm{c}}^{\pi}$. For given leading order $\ell$ the maximal (critical) trailing order is thus $k_{\mathrm{c}}(\ell)=-\ln \varepsilon_{\circ} / \ln \beta-\ell \ln \alpha / \ln \beta$. With $a=-1 / \ln \alpha$ and $b=-1 / \ln \beta$ we can introduce the maximal number of generations $L$ in subshowers with only leading branches and the maximal number of generations $T$ with only trailing branches, $L=-\ln \varepsilon_{\circ} / \ln \alpha=a \ln \varepsilon_{\circ}$ and $T=$ $-\ln \varepsilon_{\circ} / \ln \beta=b \ln \varepsilon_{\circ}$, so that the critical trailing order can be written as $k_{\mathrm{c}}(\ell)=T(1-\ell / L)$ where $\ell$ can run only between 0 and $L$. This critical condition is in Fig. 1 shown with a diagonal line. Equivalently, we can turn the condition around and express the maximal leading order given the trailing one, $\ell_{\mathrm{c}}(k)=L(1-k / T)$. It is now straightforward to write down the number of produced muons from decays of subshowers in this discrete and simplistic model. As can be seen from the thick and thin arrows crossing the diagonal decay line, the number of muons has two contributions, $N_{\mu}=$ $N_{\mu}^{(1)}+N_{\mu}^{(2)}=\sum_{\ell=0}^{\lfloor L\rfloor} N_{\mu \ell}^{(1)}+\sum_{k=0}^{\lfloor T\rfloor} N_{\mu k}^{(2)}=\sum_{\ell=0}^{\lfloor L\rfloor}\left(\begin{array}{c}\ell+\left\lfloor k_{\mathrm{c}}(\ell)\right\rfloor \\ \ell\end{array}\right) p^{\ell} n_{\mathrm{ch}}^{\left\lceil k_{\mathrm{c}}(\ell)\right\rceil}+\sum_{k=0}^{\lfloor T\rfloor}\left(\begin{array}{c}\left\lfloor\ell_{\mathrm{c}}(k)\right\rfloor+k \\ k\end{array}\right) p^{\left\lceil\ell_{\mathrm{c}}\right\rceil} n_{\mathrm{ch}}^{k}$, where the first term comes from the thick arrows and the second term is from the thin arrows crossing the diagonal line. Note that all the expressions are integers and that correct conversion to the next integer has been applied for each case. Since $\lceil x\rceil=\lfloor x\rfloor+1$, we can settle the expression by using only rounded-down values, $N_{\mu}=\sum_{\ell=0}^{\lfloor L\rfloor}\left(\begin{array}{c}\ell+\left\lfloor k_{\mathrm{c}}(\ell)\right\rfloor \\ \ell\end{array}\right) p^{\ell} n_{\mathrm{ch}}^{\left\lfloor k_{\mathrm{c}}(\ell)\right\rfloor+1}+\sum_{k=0}^{\lfloor T\rfloor}\left(\begin{array}{c}\left\lfloor\ell_{\mathrm{c}}(k)\right\rfloor+k \\ k\end{array}\right) p^{\left\lfloor\ell_{\mathrm{c}}(k)\right\rfloor+1} n_{\mathrm{ch}}^{k}$. A continuous approximation of this model is obtained by replacing discrete sums with integrals and discrete variables with continuous ones, and approximating the binomial symbol with a Gaussian 
function $\left(\begin{array}{l}n \\ k\end{array}\right) \approx 2^{n} \mathscr{N}(k ; n / 2, \sqrt{n} / 2)$. More details can be found in [9]. We also notice that since $T=-\ln \varepsilon_{\circ} / \ln \beta$ and $L=-\ln \varepsilon_{\circ} / \ln \alpha$ we can say $L=T A$, where for the asymmetric HM model we can introduce a logarithmic asymmetry $A=\ln \beta / \ln \alpha$ and a shorthand $B=A /(A-1)$.

After some integration, we finally arrive at the number of muons,

$$
N_{\mu}=T\left[A n_{\mathrm{ch}}^{1-d}+p^{1-d}\right] n_{\mathrm{ch}}^{T} \frac{\exp (T(B \ln \rho+u-2 v w))}{\sqrt{2} T(A-1) w} \operatorname{erfc}((w-v) \sqrt{T}),
$$

where $u=2 B(2 B-1), v=\sqrt{2} B$, and $w^{2}=2 B(B-1)+1 / 2-\ln 2+B \ln \rho$. The muon exponent is obtained from $N_{\mu} \propto E_{\circ}^{m}$. Since $\mathrm{d} \ln \varepsilon_{\circ}=\mathrm{d} \ln E_{\circ}$ and $\mathrm{d} \ln \varepsilon_{\circ}=-\ln \beta \mathrm{d} T$ we can get the muon exponent as $m=\mathrm{d} \ln N_{\mu} / \mathrm{d} \ln E_{\circ}=\mathrm{d} \ln N_{\mu} / \mathrm{d} \ln \varepsilon_{\circ}=-(1 / \ln \beta)\left(\mathrm{d} \ln N_{\mu} / \mathrm{d} T\right)=-(1 / A \ln \alpha)\left(\mathrm{d} \ln N_{\mu} / \mathrm{d} T\right)$. While the contribution to the muon exponent from the first two factors in Eq. (2.1) is trivial to obtain, the contribution from the integral has more terms. Gathering only the $T$-dependent terms we get the muon exponent as

$$
m=-\frac{1}{A \ln \alpha}\left[\ln n_{\mathrm{ch}}+B \ln \rho+u-2 v w+\Delta m(\varepsilon)\right] .
$$

where the large first two terms are canceled by the third to give $m \lesssim 1$ and the remaining $\Delta m(\varepsilon)$ term is a small, energy-dependent deviation from the power-law. Note that the logarithmic asymmetry $A$ is a fundamental parameter of the extended Heitler-Matthews model and is a driving force behind all of the results.

\section{Mean depth of shower maximum}

The calculation of the depth of shower maximum, $X_{\max }$, is hampered by the fact that one cannot easily derive $\left\langle X_{\max }\right\rangle$ from the individual depths of maximum of sub-showers. This problem can be avoided by calculating the shower center-of-gravity in atmospheric depth

$$
\operatorname{cg}[N(X)]=\frac{\int_{0}^{\infty} X N(X) \mathrm{d} X}{\int_{0}^{\infty} N(X) \mathrm{d} X}
$$

with $N(X)$ being the number of charged particles at depth $X$. The center-of-gravity is a linear operator and, knowing the depth of the center-of-gravity, the depth of shower maximum can be calculated. The longitudinal profile of a shower is empirically well described by the Gaisser-Hillas (GH) function [11],

$$
\mathrm{GH}\left(X ; \Lambda, X_{1}, X_{\max }, N_{\max }\right)=N_{\max }\left(\frac{X-X_{1}}{X_{\max }-X_{1}}\right)^{\frac{X_{\max }-X_{1}}{\Lambda}} \exp \left(\frac{X_{\max }-X}{\Lambda}\right),
$$

where $\Lambda$ and $X_{1}$ are shower shape parameters and $N_{\max }$ the number of particles at shower maximum. After re-writing this function as Lambert $\mathrm{W}$ function [12] one gets

$$
\operatorname{cg}\left[\mathrm{GH}\left(X ; \Lambda, X_{\circ}, X_{\max }\right)\right]=X_{\max }+\Lambda .
$$

The center-of-gravity of electromagnetic showers with energy $E=\varepsilon E_{\mathrm{c}}^{\mathrm{em}}$ can be calculated within cascade theory [13] and is given by

$$
X_{\mathrm{cg}} \approx X_{\circ}\left(\ln \varepsilon+\delta^{*}\right)
$$


with $\delta^{*}$ being an energy-independent offset that depends on the shower-inducing particle $\left(\delta^{*}=1.2\right.$ for photon showers), $X_{\circ}=36 \mathrm{~g} / \mathrm{cm}^{2}$ being the EM radiation length in air, and $E_{\mathrm{c}}^{\mathrm{em}}=87 \mathrm{MeV}$ denoting the critical energy. The center-of-gravity of an electromagnetic sub-shower starting at injection depth $X_{\mathrm{h}}$ is

$$
X_{\mathrm{cg}}=X_{\mathrm{h}}+X_{\circ}\left(\ln \varepsilon+\delta^{*}\right) .
$$

The total EM shower is then the sum of the EM sub-showers from all hadronic-shower nodes $i$. The amplitude of the sum of EM sub-showers at a node point $i$ of the cascade is proportional to the total energy of the particles decaying to photons, which is the product of the number of decaying particles $N_{i}$ and their energy $E_{i}$,

$$
F(X)=\sum_{i} \varepsilon_{i}^{*} f\left(X ; X_{\mathrm{cg}, i}\right)=\sum_{i} \varepsilon_{i}^{*} f\left(X ; X_{\mathrm{h}, i}+\delta^{*}+X_{\circ} \ln \varepsilon_{i}\right),
$$

where $\varepsilon_{i}^{*}=N_{i} \varepsilon_{i}$ is the total EM energy from sub-shower node $i$ in units of $E_{\mathrm{c}}^{\mathrm{em}}$.

Since the center-of-gravity of a shower composed of sub-showers of shapes $f_{i}$ with weights $w_{i}$ is simply

$$
\operatorname{cg}\left[\sum_{i} w_{i} f_{i}(X)\right]=\frac{\sum_{i} w_{i} \operatorname{cg}\left[f_{i}(X)\right]}{\sum_{i} w_{i}}=\sum_{i} \frac{w_{i}}{\sum_{i} w_{i}} \operatorname{cg}\left[f_{i}(X)\right]
$$

we can write the center-of-gravity of the total EM shower as

$$
X_{\mathrm{cg}} \equiv \operatorname{cg}[F(X)]=\frac{\sum_{i} \varepsilon_{i}^{*}\left(X_{\mathrm{h}, i}+\delta^{*}+X_{\circ} \ln \varepsilon_{i}\right)}{\sum_{i} \varepsilon_{i}^{*}}=\left\langle X_{\mathrm{h}}\right\rangle_{*}+\delta^{*}+X_{\circ}\langle\ln \varepsilon\rangle_{*},
$$

where $\langle x\rangle_{*}=\sum \varepsilon_{i}^{*} x_{i} / \sum \varepsilon_{i}^{*}$ is the total particle energy-weighted mean of a quantity $x$. Note that $\varepsilon_{\mathrm{em}}=\sum_{i} \varepsilon_{i}^{*}$ is the total energy that is transferred into the EM shower.

Summing over all hadronic nodes $i=\{\ell, k\}$ at the depths $X_{\mathrm{h}}=\lambda_{\mathrm{h}}(\ell+k)$ is mathematically involved and will be described in detail in Ref. [9]. Here we give only the result after applying suitable approximations. To obtain a compact expression we introduce $\delta=\delta^{*}-\ln 2$ and define

$$
Y_{\mathrm{cg}}=\frac{X_{\mathrm{cg}}-X_{1}-\delta X_{\circ}}{X_{\circ}} .
$$

with $\eta=\lambda_{\mathrm{h}} / X_{\circ}$ as the ratio of interaction lengths. Then, in the high-energy limit, the center-ofgravity reads

$$
\begin{aligned}
Y_{\mathrm{cg}} & =\eta \frac{\alpha p+\beta n_{\mathrm{ch}}}{1-\left(\alpha p+\beta n_{\mathrm{ch}}\right)}+\ln \varepsilon+\frac{\alpha q \ln \alpha+\beta n_{\circ} \ln \beta}{\alpha q+\beta n_{\circ}}+\frac{\alpha p \ln \alpha+\beta n_{\mathrm{ch}} \ln \beta}{1-\left(\alpha p+\beta n_{\mathrm{ch}}\right)} \\
& =\ln \varepsilon+\frac{\eta\left(\alpha p+\beta n_{\mathrm{ch}}\right)+\alpha \ln \alpha+(1-\alpha) \ln \beta}{\alpha q+\beta n_{\circ}} .
\end{aligned}
$$

In this approximation, the depth of the center-of-gravity is proportional to the logarithm of the energy. To a very good approximation this also holds for the depth of shower maximum, see Eq. (3.3). We also confirm the prediction of the elongation rate theorem [14]. The elongation rate $D_{\mathrm{e}}=\mathrm{d}\left\langle X_{\max }\right\rangle / \mathrm{d} \ln E_{\circ}$ of a shower is expected to be $X_{\circ}$ as long as all interactions satisfy scaling, i.e. the secondary particle distributions are universal functions of the variable $\xi=E_{i} / E_{i-1}$. This condition is satisfied by construction in the Matthews-Heitler model with energy-independent parameters. 

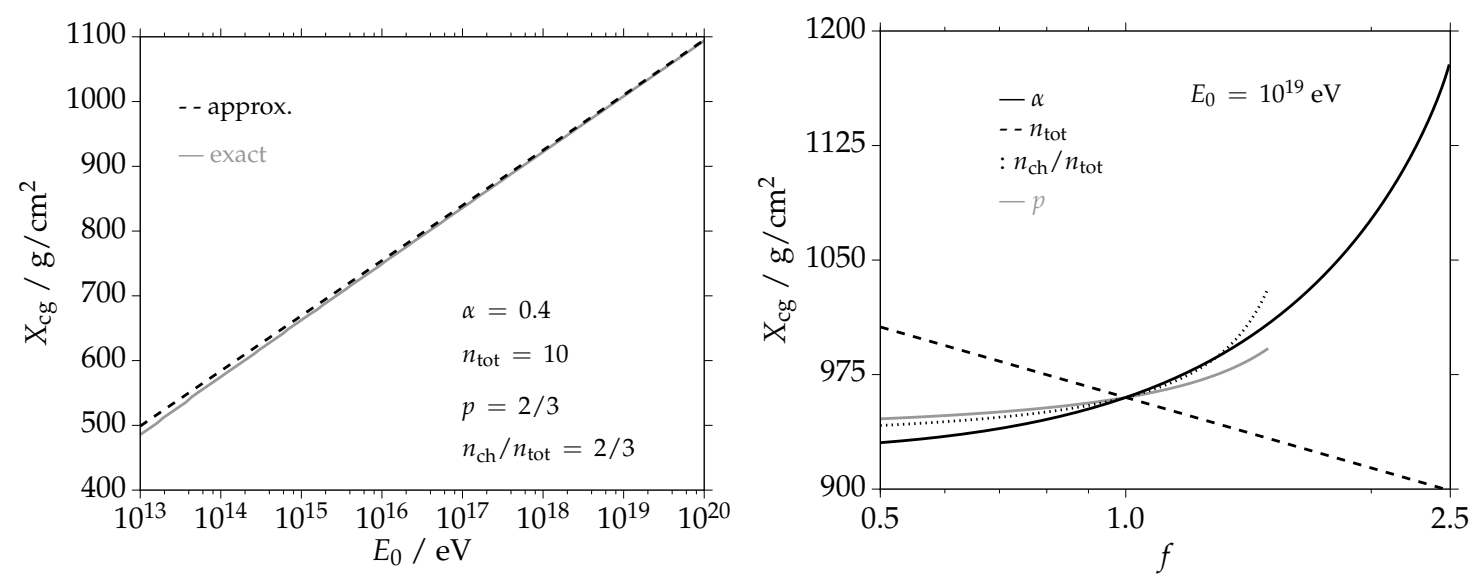

Figure 3: Left: Comparison of the depth of the center-of-gravity $T=X_{\mathrm{cg}} / X_{\circ}$ calculated by exact summation of all sub-showers and by using the high-energy approximation. Right: Dependence of the depth of the shower center-of-gravity on parameters of hadronic interactions. The parameters are scaled with a factor $f$ relative to $\alpha=0.4, n_{\mathrm{tot}}=10, n_{\mathrm{ch}} / n_{\mathrm{tot}}=2 / 3$, and $p=2 / 3$.

As a cross-check we compare the approximate result for $T=X_{\mathrm{cg}} / X_{\circ}$ with the exact summation carried our numerically. In the following diagrams we use the parameters $\alpha=0.4, p=2 / 3$, $n_{\text {tot }}=10, n_{\mathrm{ch}} / n_{\text {tot }}=: f_{\text {ch }}=2 / 3, \lambda_{\mathrm{h}}=90 \mathrm{~g} / \mathrm{cm}^{2}, X_{\circ}=37 \mathrm{~g} / \mathrm{cm}^{2}, X_{1}=90 \mathrm{~g} / \mathrm{cm}^{2}, E_{\mathrm{c}}^{\pi}=30 \mathrm{GeV}, E_{\mathrm{c}}^{\mathrm{em}}=$ $87 \mathrm{MeV}$ and $\delta^{*}=1.2$ if not otherwise indicated. The result for the high-energy approximation $E_{\circ} \rightarrow \infty$ approaches very fast that of the exact summation, see Fig. 3-left.

In Fig. 3 (right), the predictions of our model are shown for different parameters characterizing the underlying hadronic interactions. An increased elasticity strongly increases the depth of the shower center-of-gravity and an increased multiplicity decreases it. Similarly, an increase of the ratio $f_{\text {ch }}$ will shift the shower center-of-gravity to greater atmospheric depths. The results found for the analytical approximation are in good qualitative agreement with detailed numerical simulations [15].

\section{Conclusions}

We have extended the Heitler-Matthews model for extensive air showers to account for the wellknown leading particle effect in hadronic interactions. Two new parameters were introduced. One parameter describes how large an energy fraction of the projectile particle is transferred to the leading secondary hadron. The second parameter allows us to consider the case that a fraction of these leading particles will decay to electromagnetic particles before interacting. With pions being the most abundant secondary particles of hadronic interactions, the bulk of interactions in an air shower are produced by charged pions. With about a probability of $\sim 30 \%$ a charged pion will produce a leading neutral pion that will decay before interacting, except at pion energies $E_{\pi} \gtrsim$ $10^{19} \mathrm{eV}$. Hence, the possible EM decay of the leading particle is an important process that has to be considered for improving the predictions of cascade models such as the Heitler-Matthews model.

We have shown that it is possible to obtain analytic expressions for the number of muons and the mean depth of shower maximum within this model. This allows us to study analytically 
the relation between the parameters of the interaction model and general features of extensive air showers. The parameter dependences derived in the analytic model qualitatively agree with those found in detailed numerical shower simulations [15].

With respect to the number of muons, it was shown that the ratio of charged to total multiplicity and the leading particle survival probability have the largest influence on the number of muons at ground. The elasticity and the multiplicity do not strongly influence the number of muons.

Using the depth of the center of gravity of the longitudinal air shower profile as a proxy for the shower evolution, we were able to calculate predictions for the mean depth of shower maximum. A compact expression was derived in the high-energy limit. Within the extended Heitler-Matthews model presented here, the shower maximum is mainly influenced by the elasticity, the multiplicity, and the ratio of charged particles to total number of particles produced in each interaction.

\section{References}

[1] A. M. Hillas, Two interesting techniques for monte carlo simulation of very high energy hadron cascades, Proc. of 17th Int. Cosmic Ray Conf., Paris 8 (1981) 193.

[2] M. Kobal, A thinning method using weight limitation for air-shower simulations, Astropart. Phys. 15 (2001) 259-273.

[3] J. Alvarez-Muñiz, R. Engel, T. Gaisser, J. A. Ortiz, and T. Stanev, Hybrid simulations of extensive air showers, Phys. Rev. D 66 (2002) 033011, [astro-ph/ 0205302 ].

[4] H.-J. Drescher and G. R. Farrar, Air shower simulations in a hybrid approach using cascade equations, Phys. Rev. D67 (2003) 116001, [astro-ph/ 0212018 ].

[5] T. Bergmann et al., One-dimensional hybrid approach to extensive air shower simulation, Astropart. Phys. 26 (2007) 420-432, [astro-ph/ 0606564 ].

[6] J. Matthews, A Heitler model of extensive air showers, Astropart. Phys. 22 (2005) 387-397.

[7] J. M. C. Montanus, An extended Heitler-Matthews model for the full hadronic cascade in cosmic air showers, Astropart. Phys. 59 (2014) 4-11.

[8] K.-H. Kampert and M. Unger, Measurements of the Cosmic Ray Composition with Air Shower Experiments, Astropart. Phys. 35 (2012) 660-678, [arXiv: 1201.0018 ].

[9] S. Grimm, R. Engel, and D. Veberič, Extensions of the Heitler model: Leading-particle effect, in preparation, to be submitted to Astropart. Phys. (2017).

[10] J. F. Carlson and J. R. Oppenheimer, On Multiplicative Showers, Phys. Rev. 51 (1937) 220-231.

[11] T. K. Gaisser and A. M. Hillas, Reliability of the method of constant intensity cuts for reconstructing the average development of vertical showers, Proc. 15th Int. Cosmic Ray Conf., Plovdiv 8 (1977) 353-357.

[12] D. Veberič, Lambert W Function for Applications in Physics, Comput. Phys. Commun. 183 (2012) 2622-2628, [arXiv:1209.0735].

[13] B. Rossi and K. Greisen, Cosmic-ray theory, Rev. Mod. Phys. 13 (1941) 240-309.

[14] J. Linsley and A. A. Watson, Validity of scaling to $10^{20} \mathrm{eV}$ and high-energy cosmic ray composition, Phys. Rev. Lett. 46 (1981) 459-463.

[15] R. Ulrich, R. Engel, and M. Unger, Hadronic Multiparticle Production at Ultra-High Energies and Extensive Air Showers, Phys. Rev. D 83 (2011) 054026, [arXiv: 1010 . 4310]. 